künstlich unter getreuer Nachahmung der großen Lehrmeisterin Natur zu konzentrieren.

Die moderne Gesteinsuntersuchung mittels Metallmikroskops in auffallenden Licht, die kolloidchemische Forschung und die Veriolgung der Bedürfnisse der immer komplizierter werdenden Aufbereitungsmethoden sind - um nur einiges zu nennen - Mittel, um die Veredelung armer Rohstoffe vorzubereiten und somit neue Lagerstätten in den Kreis der wirtschaftlich nutzbaren Ablagerungen überführen zu helfen.

Möchte es uns dabei niemals an einsichtigen, tüchtigen und vaterländisch denkenden Männern fehlen!

[A. 186.]

\section{Die Eigenschaften des Kaliumpermanganats und das Deutsche Arzneibuch.}

\section{Von G. Fester und G. Brude, Frankfurt a. M.}

(Eingeg. 10.18. 1922.)

Es ist bemerkenswert, daß mitunter selbst bei altbekannten Stoffen ' in der Literatur sich auf fehlerhaiten Beobachtungen beruhende Angaben finden, die widerspruchslos von einem Autor zum andern übernommen werden. Ein solcher Fall liegt bei dem Kaliumpermanganat vor, und zwar hat wiederholt gerade das reinste, völlig unzersetzte Produkt eine Zurückweisung im Handel erfahren, weil seine Beschaffenheit, namentlich die Farbe, nicht mit den Literaturangaben in Einklang zu bringen ist.

Über die Frage des Permanganats finden sich bei den verschiedenen Autoren die widersprechendsten Angaben und fast an kemer Stelle sind diese - selbst unter Berücksichtigung der bei Farbangaben stets in Rechnung zu setzenden Subjektivität des Beobachters - mit den Tatsachen in Übereinstimmung $\mathrm{zu}$ bringen. Im folgenden seien einige dieser Angaben aus der Literatur aufgeführt:

M o h r: schwarz, metallglänzend.

Gmelin-Kraut: dunkelpurpurfarbene Prismen.

D a m m er: dunkle, kupfrig glänzende, dichroitische rhombische Prismen.

Muspratt: dunkelrote, fast schwarze Kristalle von grünlichem Metallglanz.

S m it h: purpurne Kristalle mit grünlichem Oberflächenglanz.

Deutsohes Arzneibuch V: dunkelviolette, fast schwarze, stahlblau glänzende, trockene Prismen.

The British Pharmacopoeia 1914: dark purple, iridescent.

U. S. Pharmacopoei a IX: dark purple colour, almost opaque by transmitted light and of a blue metallic luster by reflected light.

Durch eine Anzahl einfachster Versuche kann man sich davon überzeugen, daß die meisten dieser Angaben, insbesondere die des Deutschen und Amerikanischen Arzneibuches, für reines Permanganat unzutreffend sind oder unzulässigerweise die Pulverfarbe auf die Kristalle übertragen.

Versuch 1. Reines Kaliumpermanganat des Handels wurde aus Wasser umkristallisiert und im Dunkeln in reiner Luft (nicht im Laboratorium) auf einem Tonteller getrocknet. Die Farbe wird am besten als Braun, mit metallischem Oberflächenglanz, Bronzeglanz, bezeichnet. Bei sehr kleinen Kristallen erscheint die Farbe dunkler, fast schwarz, doch ist niemals eine violette Tönung festzustellen. Erst wenn die Kristalle zu ganz feinem Pulver zerrieben werden, tritt eine dunkelviolette Farbe auf, wie sie auch der Strich auf unglasiertem Porzellan zeigt. Nach den Feststellungen von R $\theta$ tgers ${ }^{1}$ ) zeigen lediglich mikroskopisch dünne Nadeln im durchfallenden Licht rote Farbe ohne besonderen Pleochroismus.

Versuch 2. Die nach 1. erhaltenen Kristalle wurden offen im Laboratorium im gedämpften Tageslicht liegengelassen. 'Nach einigen Stunden war ideutlich eine violette Tönung festzustellen, und nach zwei Tagen zeigten die Kristalle stahlblauen Oberflächenglanz. Diese Veränderung betraf nur die oberste Schicht, darunter blieb die ursprïngliche braune Farbe länger erhalten, welche auch die frischen Bruchflächen zeigten. Kristalle, die im Laboratorium im Dunkeln liegengelassen wurden, zeigten die Anlauffiarben erst nach längerer Teit.

Versuch 3. Versuch 2 wurde in reiner Luft außerhalb des Laboratoriums wiederholt. Die Anlauffarben traten auch hier auf, doch erheblich langsamer als bei 2. Sehr rasch dagegen war die Einwirkung im direkten, nicht durch Glas geschwächten Sonnenlicht; in diesem Fall war schon nach einem Tag die stahlblaue Farbe wahrzunehmen. Im großen tritt in Glasgefäßen und Fässern im Verlauf von einigen Wochen ebenfalls eine allmähliche Verfärbung ein, die von oben nach unten fortschreitet.

Versuch 4. Frisch bereitete Kristalle wurden auf zwei Erlenmeyerkolben verteilt, die, nachdem der eine teilweise mit Kohlendioxyd angefüllt war, zugekorkt wurden. Nach einem Tag war unter der Einwirkung der Kohlensäure ebentalls eine Verfärbung nach Violett zu beobachton.

Versuch 5. Kristalle von frisch bereitetem Permanganat wurden auf dem Boden eines Erlenmeyerkolbens ausgebreitet und dann ein mit konzentrierter Salzsäure gefülltes Reagenzglas schräg ein-

1) Ztschr. f. phys. Chem. 8, 14 [1891]. gelegt. Sofort trat eine in konzentrischen Kreisen fortschreitende Verfärbung von Braun über Violett und Stahlblau in Graphitschwarn ein, das nach einiger Zeit in stumples Braunschwarz überging.

Zusammenfassend läßt . sich also sagen, daß die ursprüugliche bronzebraune Farbe der Permanganatkristalle infolge oberflächlicher Zersetzung durch den Kohlendioxydgehalt der Luft und wohl auch organischen Staub über Violett in Stahlblau übergeht; die oberste Lage wirkt dabei gewissermaßen als Filter und schützt die darunterliegenden Schichten. Sehr raseh tritt die Verfärbung in mineralsäurehaltiger Luft ein und wird ferner durch das Licht besonders beschleunigt. Die Angaben der Literatur, namentlich der Arzneibücher, sind also dementsprechend zu korrigieren. Die Bezeichnung. in der Deutschen Pharmakopöe würde wohl am zweckmäßigsten so zu formulieren sein: "Trockene bronzebraume Kristalle, mit metallischem Glanz, beim Liegen an der Luft violette bis stahlblaue Anlauffarben annehmend."

Auch hinsichtlich der Kristallforr." iild die Angaben des Arzneibuches zweckmäßig abzuändern. Es findet sich lediglich die $\mathrm{Be}$ zeichnung "Prismen“, was kristallographisch richtig ist, aber, da hierunter im vulgären Sinne ausschließlich langgestreckte Kristallindividuen verstanden werden, doch zu Irrtümern Veranlassung geben kainn. Vielfach kommen im Handel - je nach den Bedingungen der Kristallisation im Großbetrieb - auch kurzprismatische, fast isometrische Kristalle vor, und es erscheint deshalb zweckmäßiger, den auf alle Fälle passenden Ausdruck ,rhombisch-bipyramidale" oder bloß "rhombische Kristalle“ zu wählen.

Endlich ist auch" noch die Angabe des Arzneibuches völlig irreführend, daß eine Lösung $1+999$ Lackmuspapier nicht verä̉ndern darf und steht auch im Gegensatz zu der späteren Feststellung des gleichen Abschnittes, daß die Lösung durch Weingeist und andere reduzierende Stoffe entfärbt wind. Tatsächlich wird eine Permanganatlösung durch die meisten organischen Stoffe, namentlich durch Papier und auch durch den Lackmusfarbstoff selbst reduziert, wobei dann unter Ausscheidung von Mangandioxyd freies Alkali gebildet wird. Bringt man einen Tropfen reinster Permanganatlösung auf rotes Lackmuspapier, so bildet sich je nach der Bereitung des Reagenzpapieres in kürzerer oder längerer Zeit ein brauner Fleck, der von einem blauen Hof umgeben ist. Die diesbezügliche Vorschrift des Arzneibuches müBte also bei der bevorstehenden Neuauflage ganz in Wegfall kommen.

[A. 204.]

\section{Berichtigung:}

Im Aufsatz Kropf: „Zur kolorimetrischen Vanadinbestimmung in Stahlen" (Ang. Chem. 35, 366 [1922], r. Sp.) Abschnitt: Durchführung der Bestimmung, 9. Zeile v. o. muß es heißen: ...oxydiert man mit je $1 \mathrm{ccm}$ Salpetersäure $(1,18) \ldots$ statt ,... mit je $1 \mathrm{ccm}$ Schwefelsäure."

on.

\section{Rundschau.}

Während der Herbstmesse in Frankfurt a. M. (8.-14. Okt.) wird ein Tag der Technik" (10. Oktober) stattfinden. Am Vorabend, 9. Oktober, wird mit verbindendem Vortrag des Direktors Schröder der ColonnaFilmgesellschaft, die den Einstein-Film herausgebracht hat, eine Auswahl von Werbe- und Industriefilmen zur Vorführung kommen. Am Dienstag, 10. Oktober, spricht Prof. Dr. E b erle (Darmstadt) tuber, Wärmeund Elektrowirtschaft". Danach wird das "Haus der Technik" der Frankfurter Messe besichtigt. Am Nachmittag werden unter anderm Generaldirektor Dr. Bergius, Prof. Fester, Prof. Ruppel, Direktor Dr. Vogelsang sprechen. Der Verband Deutscher Diplomingenieure beruft einen Gautag seiner südwestdeutschen Bezirksvereine. Abends 6 Uhr hält der vor kurzem von seinen wissenschaftlichen und Vortragsreisen nach Spanjen und Amerika zurückgekehrte Professor für physikalische Grundlagen der Medizin, Dr. Friedrich Dessauer, einen Festvortrag über „Kulturwerte der Technik“". Für Mittwoch, 11. Oktober, sind Exkursionen nach Gustavsburg (Werk der Maschinenfabrik Angsburg-Nümberg), nach Nied (Eisenbahnwerkstätte), nach Kesselstadt (Mainkraftwerk) sowie Besichtigung führender Frankfurter Industrieunternehmungen und der Hauptbahnhofserweiterung in Aussicht genommen. Auskünfte und Teilnehmerkarten sind erhältlich beim „Ausschuß zur Vorbereitung des Tages der Technik während der Herbstmesse Frankfurt 1922" (Haus Offenbach, Frankfurt a. M.)

\section{Neue Bücher.}

Leitfaden für Eisenhüttenlaboratorien. Von Ledebur. Elfte Auflage. neu bearbeitet von $H$. Kinder, Chefchemiker der Rhein. Stahlwerke zu Duisburg-Meiderich, und Dr.-Ing. A. Stadeler, Laboratoriumsvorstand der Henrichshütte zu Hattingen-Ruhr. 182 Seiten mit 21 Abbildungen. Verlag Friedr. Vieweg \& Sohn, A.-G. Braunschweig 1922. Preis geh. M 168, geb. M 240

Der bekannte Leitfaden von Ledebur hatte 9 Auflagen erlebt. Nach dem Tode Led eburs war es fraglich, ob das Buch in die richtigen Hände kommen würde, die dem Buche den Ruf als zuverlässiger Ratgeber in allen einschlägigen Fällen erhalten könnten. Durch einsichtige Vermittlung des Vereins deutscher Eisenhüttenleute wurde die 